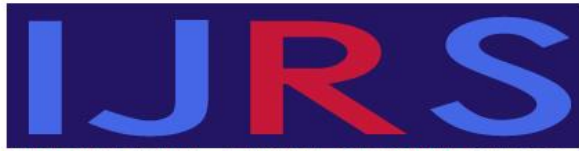

Hipatia Press

www.hipatiapress.com

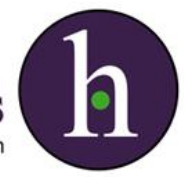

Instructions for authors, subscriptions and further details:

http://ijrs.hipatiapress.com

\title{
Diseñando e Implementado Políticas Públicas con y para la Comunidad Gitana. El Impacto Social del Plan Integral del Pueblo Gitano en Cataluña
}

Tania García-Espinel ${ }^{1}$, Dolores Santiago-Santiago ${ }^{2}$, and Manuel García-Algar ${ }^{3}$

1) Departamento de Trabajo, Asuntos Sociales y Familias, Generalitat de Catalunya. España

2) Universitat Rovira y Virgili. España

3) CampusRom, Red Gitana Universitaria de Catalunya. España

Date of publication: March $15^{\text {th }}, 2019$

Edition period: March 2019- September 2019

To cite this article: García-Espinel, T., Santiago-Santiago, D., y GarcíaAlgar, M. (2019). Diseñando e implementando políticas públicas con y para la comunidad gitana. El impacto social del Plan Integral del Pueblo Gitano en Cataluña. International Journal of Roma Studies, 1(1), 84-119. doi: 10.17583/ijrs.2019.3957

To link this article: http://dx.doi.org/10.17583/ijrs.2019.3957

PLEASE SCROLL DOWN FOR ARTICLE

The terms and conditions of use are related to the Open Journal System and to Creative Commons Attribution License (CCAL). 


\section{Designing and Implementing Public Policies with and for Roma People. The Social Impact of the Integrated Plan for the Roma in Catalonia}

Tania García Espinel

Generalitat de Catalunya

Manuel García Algar

CampusRom
Dolores Santiago

Universitat Rovira i Virgili

\section{Abstract}

Since 2015 when the European Parliament recognized the racial discrimination and social exclusion suffered by the Roma community, a growing political effort is being done at European, national, regional and local level promoting their inclusion. However, the social and especially the educational situation of this community remains alarming. Based on the results obtained in the framework of two scientific investigations (GarcíaEspinel, 2016, Macías-Aranda, 2017), this article demonstrates the key principles that have made possible the impact of the Integral Plan for the Roma People in Catalonia. Different elements have been identified as key in making possible the social impact of this Plan. Important improvements in both education (mandatory and post-compulsory) and in the living conditions of Roma children and adults are some of the advancements achieved by this policy in Catalonia.

Keywords: Roma People, Public Policies, Educational Success, Antigypsyism, Public Participation 


\section{Diseñando e Implementando Políticas Públicas con y para la Comunidad Gitana. El Impacto Social del Plan Integral del Pueblo Gitano en Cataluña}

Tania García Espinel

Generalitat de Catalunya

Manuel García Algar

CampusRom
Dolores Santiago

Universidad Rovira $i$ Virgili

\section{Resumen}

Desde que en 2005 el Parlamento Europeo reconociera la discriminación racial y exclusión social que sufre la comunidad gitana, se está llevando a cabo un creciente esfuerzo político a nivel europeo, estatal, regional y local en favor de su inclusión. Sin embargo, la situación social y especialmente educativa de esta comunidad sigue siendo claramente alarmante. A partir de los resultados alcanzados en el marco de dos investigaciones científicas (García-Espinel, 2016; Macías-Aranda, 2017), este artículo evidencia los principios clave que han posibilitado el impacto del Plan Integral del Pueblo Gitano en Catalunya. Son diversos los elementos clave que están haciendo posible el impacto social de este Plan. Entre los avances de esta política en Catalunya destacan importantes mejoras en educación (obligatoria y postobligatoria) y en las condiciones de vida de niños y adultos gitanos.

Palabras clave: Pueblo Gitano, Políticas Públicas, Éxito Educativo, Antigitanismo, Participación Pública 


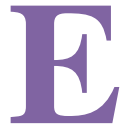

1 jueves 28 de abril de 2005, en Bruselas, el Parlamento Europeo adoptó una Resolución sin precedentes sobre la situación de la población romaní en la Unión Europea (Parlamento Europeo, 2005). En dicho texto, motivado principalmente por la lucha de la sociedad civil gitana y por los resultados de la investigación científica (Aiello, Mondejar, \& Pulido, 2013), las instituciones europeas reconocen, por primera vez, que la población gitana de Europa sufre discriminación racial y graves discriminaciones estructurales, pobreza y exclusión social, así como una discriminación múltiple. Por ello, de manera histórica, el Parlamento Europeo pide al Consejo, a la Comisión, a los Estados miembros y a los países candidatos que consideren el reconocimiento de la población romaní como minoría europea. Uno de los países que reconocerá al Pueblo Gitano como una minoría nacional es España, a través de una Proposición no de ley relativa al reconocimiento de los derechos del Pueblo Gitano en España (Congreso de los Diputados, 2005). La Resolución europea de 2005 también considera, entre otros, que el holocausto de la población romaní merece un reconocimiento pleno y proporcional a la gravedad de los crímenes nazis concebidos para exterminar físicamente a la población romaní de Europa (Samudaripen).

Desde entonces, se han desarrollado numerosas recomendaciones e iniciativas políticas en favor de la inclusión del Pueblo Gitano, transformando el marco europeo, estatal, regional y local de numerosas políticas públicas que se diseñan sobre (o para) esta comunidad. Desde el propio Parlamento Europeo, el 28 de enero de 2008, se adoptaba una propuesta de Resolución común (iniciada por varias fuerzas políticas en 2004 y finalizada en 2009) sobre una estrategia europea relativa a la población romaní (European Parliament, 2008). Esta resolución motivó al mismo organismo europeo a adoptar en 2011 una resolución similar, pero más concreta, incluyendo áreas prioritarias y objetivos específicos la estrategia europea romaní.

Este posicionamiento hacia los derechos del Pueblo Gitano desde el Parlamento Europeo se debe considerar, sin duda, como el impulso o precedente del actual marco político europeo en favor de la comunidad gitana, ya que ha estimulado el desarrollo de políticas específicas sobre el 
Pueblo Gitano, tanto en el seno de la Comisión Europea como en los estados miembros.

En virtud de ello, destacan el marco europeo de estrategias nacionales de inclusión de los gitanos hasta 2020 (European Commission, 2011a), adoptado en abril de 2011 por la Comisión Europea con el objetivo de definir y coordinar las obligaciones y responsabilidades de los Estados miembros de la UE y los países candidatos con respecto a sus poblaciones gitanas. Dentro de este Marco, la Comisión plantea que los Estados miembros deben desarrollar sus propias estrategias nacionales gitanas, desde cuatro objetivos clave para la inclusión social (vivienda, salud, educación y empleo), y basándose en los 10 principios básicos comunes para la inclusión de los gitanos (Comisión Europea, 2009) y en los principios del Decenio de la Inclusión Gitana (Brüggemann \& Friedman, 2017), entre otros.

Este marco europeo ha provocado que todos los estados miembros de la UE tengan actualmente una estrategia nacional gitana, como sucede con el estado español, donde se desarrolla la Estrategia nacional para la inclusión social de la población gitana en España 2012-2020 (Ministerio de Sanidad. Servicios Sociales e Igualdad, 2012). Para alcanzar los objetivos de dicha Estrategia nacional, el estado español ha elaborado diferentes Planes Operativos (2014-2016 y 2018-2020) que pretenden ofrecer orientaciones a los actores implicados en el desarrollo e implementación de la estrategia nacional, así como pautas y medidas específicas de cada ámbito prioritario de actuación (Ministerio de Sanidad. Servicios Sociales e Igualdad, 2013, 2018).

Sin embargo, a pesar de los numerosos esfuerzos políticos en favor de la inclusión del Pueblo Gitano, la situación social y educativa de esta comunidad sigue siendo claramente alarmante.

Según el Informe FOESSA, el 33,3\% de los hogares gitanos sufren pobreza moderada y el $37,8 \%$ pobreza grave, mientras que entre el resto de la población la pobreza moderada afecta al 20,6\% de los hogares (menos de 13 puntos) y la pobreza grave el 6,5\% (menos de 30 puntos) (Damonti \& Arza, 2014).

Si nos centramos en el ámbito educativo, del casi millón de gitanas y gitanos que se estima viven es España (Ministerio de Sanidad. Servicios 
Sociales e Igualdad, 2012), únicamente un $1 \%$ ha conseguido finalizar estudios universitarios (Ministerio de Sanidad. Política Social e Igualdad, 2011), mientras que casi un 35\% del resto de la población posee estudios superiores (INE, 2017). Esta enorme brecha educativa se observa también durante la educación obligatoria. Sólo el 30\% del alumnado gitano obtiene el graduado de educación secundaria, cuando el $60 \%$ del alumnado no gitano supera con éxito esta etapa (Ministerio de Sanidad. Política Social e Igualdad, 2011; Ministerio de Sanidad. Servicios Sociales e Igualdad, 2016).

La segregación del alumnado gitano en aulas de bajo rendimiento, las adaptaciones curriculares basadas en un curriculum de mínimos, los estereotipos y prejuicios sobre el alumnado gitano y sus familias, así como numerosas prácticas educativas basadas en ocurrencias y no en las recomendaciones de la comunidad científica, son las principales causas de la exclusión educativa del Pueblo Gitano (Consejo de la Unión Europea, 2013; Macías-Aranda \& Flecha, 2013; Santiago \& Maya, 2012; Vargas \& Gómez, 2003).

Si observamos a la comunidad gitana en el resto de ámbitos sociales, nos encontramos, nuevamente, con una situación muy desfavorable con respecto al resto de la población española: mayores índices de desempleo, menor esperanza de vida y mayor mortalidad infantil, notables desigualdades en el estado de salud, así como en el acceso a los servicios sanitarios, o significativos problemas en el acceso a una vivienda digna, así como otros problemas relacionados con la vivienda, como la segregación residencial (Damonti \& Arza, 2014; EU-FRA, 2012, 2014a, 2014b, 2014c; Ministerio de Sanidad. Política Social e Igualdad, 2011; Ministerio de Sanidad. Servicios Sociales e Igualdad, 2016). Todas estas desigualdades afectan con mayor intensidad a las mujeres gitanas, ya que ellas sufren una triple discriminación: por ser gitanas, mujeres (de igual modo que el resto de mujeres del conjunto de la sociedad) y porque frecuentemente no han accedido a una formación académica de calidad (EU-FRA, 2014a; Fernández, Domínguez, \& Flecha, 2004; García-Espinel, 2015; MacíasAranda \& Redondo, 2012).

Especialmente preocupante es el aumento del Antigitanismo, la forma específica de racismo contra el Pueblo Gitano ya reconocida 
internacionalmente por numerosos organismos e instituciones (Council of Europe, 2012a, 2012b; ECRI, 2011; European Parliament, 2015). El reciente informe de la Agencia de los Derechos Fundamentales de la Unión Europea (EU-FRA, 2018) evidencia que las formas más atroces de Antigitanismo, el crimen motivado por el odio y el acoso, siguen obstaculizando la inclusión de las personas gitanas en Europa. Según la EUFRA, a pesar de varios años de esfuerzos de inclusión, en promedio, uno de cada tres gitanos encuestados había sufrido algún tipo de acoso (comentarios ofensivos o amenazantes en persona, amenazas de violencia en persona, gestos ofensivos o miradas inapropiadas, ofensivos o amenazantes correos electrónicos o mensajes de texto, o comentarios ofensivos sobre ellos en Internet). Lo que es más preocupante para la EUFRA es que el cuatro por ciento de las personas gitanas encuestadas experimentó violencia física motivada por el Antigitanismo, pero sólo uno de cada tres lo denunció a alguna organización, incluida la policía. La EUFRA alerta de que no se está prestando la suficiente atención a las manifestaciones de Antigitanismo que acontecen en la actualidad, lo que disminuye la confianza de los y las romaníes en las instituciones, y muy particularmente en la ley, las políticas y la justicia (EU-FRA, 2018).

No obstante, y aunque quizás demasiado lentamente, cada vez son más las iniciativas políticas que están consiguiendo tener un evidente impacto social en la mejora de las condiciones de vida de la comunidad gitana. Además, este impacto social está llegando acompañado del aumento de la participación social del Pueblo Gitano en el diseño y en la implementación de dichas políticas públicas.

En este sentido, este artículo evidencia el impacto social del Plan Integral del Pueblo Gitano en Catalunya; un plan interdepartamental desarrollado por la Generalitat de Catalunya que recoge el conjunto de políticas públicas que se diseñan e implementan específicamente en favor de la inclusión de la comunidad gitana de Catalunya. La investigación desarrollada pone de manifiesto evidencias de mejora en la vida de las personas gitanas y los puntos clave que explican el impacto social de dicho Plan, y demuestra cómo se pueden diseñar e implementar políticas públicas con y para la comunidad gitana. Todo ello está permitiendo el éxito social y 


\section{García-Espinel, et al. - Plan Integral Pueblo Gitano Catalunya}

educativo de esta comunidad, además de luchar contra el Antigitanismo, superando los estereotipos y prejuicios que se asocian al Pueblo Gitano.

\section{Antecedentes del Plan Integral del Pueblo Gitano en Catalunya}

Tal y como se ha indicado al inicio de este artículo, Europa reconoce de manera oficial al Pueblo Gitano como una minoría étnica en el año 2005, y empieza a promover el desarrollo europeo y nacional de políticas públicas en favor de la comunidad gitana de manera explícita a partir del año 2008, y de manera más concreta en 2011. En este sentido, y teniendo en cuenta estos hechos y fechas tan importantes para la comunidad gitana, Catalunya debe considerarse pionera en la lucha por la igualdad y los derechos del Pueblo Gitano. En 2001 el Parlamento de Catalunya adoptaba dos Resoluciones que marcarían un antes y un después en el diseño de políticas públicas dirigidas a la comunidad gitana.

La primera de estas Resoluciones fue la 1045/VI, de 21 de noviembre de 2001, sobre la elaboración de un plan integral del Pueblo Gitano en Cataluña, bajo la cual el Parlamento catalán instaba al Gobierno de Catalunya, entre otros, a elaborar un estudio sobre la población gitana en Cataluña, con la colaboración de las entidades gitanas, como base para la elaboración de un plan integral del Pueblo Gitano en Cataluña. Esta Resolución demuestra que Catalunya empezó a promover el diseño de políticas públicas en favor de la comunidad gitana siete años antes que el Parlamento Europeo.

Por lo que respecta a la segunda Resolución, esta fue la 1046/VI, de 21 de noviembre de 2001, sobre el reconocimiento de la identidad del Pueblo Gitano y del valor de su cultura. Al amparo de esta resolución, por primera vez en la historia, un Parlamento reconocía la identidad del Pueblo Gitano y el valor de su cultura como salvaguarda de la realidad histórica de este pueblo, e instaba a un gobierno (en este caso al de Catalunya) a realizar las gestiones pertinentes para contribuir a difundir el reconocimiento de la cultura gitana y del valor de esta para la sociedad catalana. Este reconocimiento se produjo en Catalunya, pues, cuatro años antes de que se produjera en el Parlamento Europeo o en el Congreso de los Diputados de España. 
Este marco político sin precedentes permite obtener en 2003 un estudio sobre la población gitana en Cataluña, elaborado por encargo del Departamento de Bienestar y Familia de la Generalitat de Cataluña. El objeto del estudio fue obtener un conocimiento general de la situación del Pueblo Gitano en Cataluña con el fin de disponer de datos actualizados y pautas orientativas para la planificación de acciones políticas futuras, contando con la colaboración de las entidades gitanas de Cataluña, así como con la sociedad civil gitana en general o con otras estructuras de gran influencia dentro de esta comunidad, como la Iglesia Evangélica de Filadelfia. Este estudio de corte mixto, en el que se obtuvieron datos tanto cualitativos como cuantitativos, se basó en una muestra de más de 1000 personas gitanas de toda Catalunya, y su equipo de trabajo estuvo compuesto por personas gitanas y no gitanas, hombres y mujeres, jóvenes y mayores, interactuando desde la experiencia de trabajo acumulado desde las organizaciones gitanas y desde el mundo académico. Por primera vez, un estudio elaborado por encargo de la administración pública de Catalunya evidenciaba las problemáticas o barreras que encuentra la población gitana en áreas fundamentales como la educación, el empleo, la vivienda o la salud (Departament de Benestar i Família. Generalitat de Catalunya, 2005), lo que fue crucial para el diseño del primer Plan del Pueblo gitano de Catalunya.

Ateniendo a los resultados de dicho estudio, y a la luz de las Resoluciones del Parlamento de Catalunya de 2001, el Gobierno catalán elaboró el primer Plan Integral del Pueblo Gitano en Cataluña (2005-2008). El objetivo general del plan fue combatir desde una perspectiva global las barreras educativas, laborales, económicas y de carácter diverso que sufre la población gitana en Cataluña para que se pueda equiparar sociocultural y económicamente a la sociedad catalana de la que forma parte y, al mismo tiempo, promover y reconocer los rasgos culturales del Pueblo Gitano. En base a los objetivos generales de este primer plan, cada uno de los Departamentos del Gobierno de Catalunya implicados elaboraron propuestas de aplicación en su ámbito sectorial, temporalizadas y cuantificadas presupuestariamente, con el fin de mejorar la situación de la comunidad gitana. Entre las medidas específicas planteadas en este primer Plan Integral del Pueblo Gitano hubo desde la promoción de la 


\section{García-Espinel, et al. - Plan Integral Pueblo Gitano Catalunya}

recuperación de su lengua propia y común, que es el romanó, y el apoyo a la creación de programas de sensibilización a implantar en el sistema educativo, hasta planes específicos de promoción y formación para la mujer gitana o actuaciones de rehabilitación de viviendas y erradicación del chabolismo, además de medidas de inserción socio laboral.

Desde ese primer Plan, el Gobierno de Catalunya ha aprobado tres ediciones más: 2009-2013, 2014-2016 y 2017-2020. Es importante destacar que todos estos Planes han tenido un marcado carácter interdepartamental, es decir, han implicado a prácticamente todos los Departamentos del gobierno catalán, los cuales, a través del Plan integral del Pueblo Gitano, desarrollan medidas específicas en favor de la mejora de la situación de la comunidad gitana. Entre los departamentos implicados destacan los que tienen competencias en materia de presidencia, educación, vivienda, salud, trabajo, justicia, interior o cultura, entre otros. Cada uno de estos planes tuvo como punto de salida los resultados del Plan que les predecía, incorporado así importantes mejoras en su diseño, implementación y evaluación. Tanto es así, que, la Agencia de la Unión Europea por los Derechos Fundamentales selecciona en 2009 al Plan Integral del Pueblo Gitano de Catalunya como la única iniciativa positiva de carácter político y regional en favor de la comunidad gitana. En palabras de la propia Agencia:

España es, de todos los países investigados, el que ofrece el mejor ejemplo de un plan estratégico de ámbito regional para mejorar la situación de la población gitana procedente de otros Estados miembros. (...) En el ámbito regional, el Plan integral del pueblo gitano en Cataluña se considera un modelo de buena práctica en cuanto a la planificación estratégica de una administración regional para la integración de la población gitana (...). El Plan catalán es el único, de todos los países investigados, que puede ponerse como ejemplo de integración explícita de las cuestiones que afectan a la población gitana (...) (p. 8)

\section{Metodología}

Los resultados que aquí se presentan se han obtenido a partir de dos investigaciones científicas. La primera de ellas (2013-2016), desarrollada 
en la Universidad de Granada, tuvo como objetivo principal demostrar el impacto político alcanzado gracias a la nueva línea de investigación europea con y para el Pueblo Gitano, y cómo esta nueva línea permitió la aparición y el impacto social del Plan Integral del Pueblo Gitano en Catalunya (García-Espinel, 2016). La segunda investigación (2014-2017), llevada a cabo en la Universidad de Barcelona con financiación del Ministerio de Economía y Competitividad del Gobierno de España, tenía como objetivo principal el estudio y análisis del proceso a través del cual el Pueblo Gitano se convierte en un agente activo y protagonista de su propia inclusión social gracias a su participación en Actuaciones Educativas de Éxito (Flecha, 2015), realizando así importantes contribuciones científicas (teóricas y prácticas) para luchar contra la pobreza y el Antigitanismo que padece gran parte de esta comunidad (Macías-Aranda, 2017).

Ambas investigaciones se han desarrollado bajo las orientaciones y principios de la metodología comunicativa. Esta metodología de investigación se utiliza para el análisis de fenómenos, situaciones o interacciones sociales, incidiendo en los componentes que generan exclusión social y los que contribuyen a superarla (Pulido, Elboj, Campdepadrós, \& Cabré, 2014), aportando así soluciones efectivas para abordar las necesidades y problemáticas reales que afectan a los grupos sociales, especialmente a los más vulnerables (Gómez, Puigvert, \& Flecha, 2011). La metodología comunicativa se basa principalmente en el diálogo intersubjetivo y en una relación igualitaria entre el equipo de investigación y las personas participantes (Gómez, Racionero, \& Sordé, 20102, quienes participan en un plano de igualdad durante todas las fases de la investigación (Puigvert, Christou, \& Holford, 2012). De hecho, el Pueblo Gitano demanda una intervención e investigación de utilidad social, en la que se cuente con su voz y su participación y que responda a las necesidades reales de esta comunidad para así lograr mejorar su situación social, más allá de los meros diagnósticos y de las investigaciones reproduccioncitas basadas en prejuicios y estereotipos sobre esta comunidad (Amador, 2016; Hancock, 1988; Macías-Aranda \& Redondo, 2012; Vargas \& Gómez, 2003). Dada la naturaleza de la metodología comunicativa y su gran utilidad social en la intervención e investigación con y para los grupos más vulnerables, esta metodología ha sido 
recomendada por la Unión Europea por su potencial impacto social (The European Union Spanish Presidency, 2010).

Concretamente, para este artículo se recogen los resultados obtenidos a través de dos estudios de caso, realizados cada uno ellos en una de las investigaciones anteriormente mencionadas, y llevados a cabo en el mismo escenario: el Plan Integral del Pueblo Gitano de la Generalitat de Catalunya. En sendos estudios de caso se han utilizado técnicas cualitativas de orientación comunicativa. En total, este articulo utiliza evidencias recogidas de un total de 60 técnicas de recogida de la información, distribuidas de la siguiente manera:

- Análisis documental de 22 fuentes secundarias (memorias, actas, documentos internos de seguimiento y evaluación, e informes de la administración catalana).

- 13 entrevistas en profundidad de orientación comunicativa realizadas a:

- 5 personas profesionales (4 gitanas y 1 no gitana) implicadas directamente en la definición y/o implementación del Plan Integral del Pueblo Gitano en Catalunya;

- 2 decisores políticos (no gitanos);

- 2 personas gitanas destinatarias de alguna de las políticas públicas definidas desde el Plan Integral del Pueblo Gitano en Catalunya;

- 4 miembros de la comunidad científica vinculados a la investigación científica con y para el pueblo gitano (2 personas investigadoras gitanas, y 2 no gitanas).

- 11 relatos comunicativos de vida cotidiana a personas gitanas destinatarias de alguna de las políticas públicas definidas desde el Plan Integral del Pueblo Gitano en Catalunya;

- 1 grupo de discusión realizado con 4 personas gitanas destinatarias de alguna de las políticas públicas definidas desde el Plan Integral del Pueblo Gitano en Catalunya.

- y 12 observaciones realizadas en los siguientes espacios:

- Actuación Educativa de Éxito - Formación de Familiares (7 observaciones): 
- Grupo de Acceso a la Universidad del Plan Integral del Pueblo Gitano (5 observaciones);

- Grupo de Graduado en Secundaria del Plan Integral del Pueblo Gitano (2 observaciones);

- Órganos de participación del Plan Integral del Pueblo Gitano (2 observaciones);

- Reuniones del equipo de redacción del Plan Integral del Pueblo Gitano (2 observaciones);

- Actuación Educativa de Éxito - Tertulia Literaria Dialógica (2 observaciones):

- Tertulia literaria dialógica desarrollada en una de las escuelas prioritarias del Plan Integral del Pueblo Gitano (2 observaciones)

Es de especial relevancia destacar que, excluyendo las observaciones comunicativas y el análisis documental de fuentes secundarias, de las 28 personas que han participado en el resto de técnicas, 23 eran personas gitanas y 5 no gitanas, lo que supone un $82 \%$ de personas gitanas participantes en la muestra, frente al $18 \%$ de personas no gitanas.

Finalmente, es importante señalar que el análisis de la información se ha realizado teniendo en cuenta los principios de la metodología comunicativa, lo que nos ha permitido evidenciar que elementos están contribuyendo a mejorar la situación social de la comunidad gitana, haciendo especial énfasis en el impacto social y los elementos clave de las políticas públicas dirigidas en favor de la inclusión de esta comunidad.

A continuación, se exponen los elementos que explican el impacto conseguido por el Plan Integral del Pueblo Gitano en Catalunya, y que lo diferencian claramente de otros planes similares, así como evidencias cuantitativas y cualitativas de dicho impacto social.

\section{Principios clave para comprender el impacto social del Plan Integral del Pueblo Gitano en Catalunya}

Si bien es cierto que los objetivos de los diferentes Planes integrales del Pueblo Gitano en Catalunya han ido evolucionando desde el primer Plan 
(2005-2008) hasta el actual cuarto Plan (2017-2020), podemos afirmar que a partir del tercer Plan (2014-2016), y muy especialmente a partir del cuarto Plan (2017-2020), se observan unos principios o características clave que explican el impacto social que se está alcanzado, por las acciones que se enmarcan bajo dichos Planes, y que se evidenciarán en el siguiente apartado.

En primer lugar, uno de estos principios clave hace referencia a la implicación de la población gitana en el diseño, desarrollo e implementación del Plan Integral. Tanto la Comisión Europea como el Consejo de Europa reclaman que se asegure la participación activa e informada de representantes del Pueblo Gitano en todas aquellas políticas y acciones que les afectan directamente (European Commission, 2011c; Council of Europe, 2012a). También la investigación científica viene evidenciando desde hace años la importancia e impacto que supone involucrar activamente y de un modo igualitario a la población gitana en el diseño, ejecución y evaluación de la investigación científica y la intervención social (Flecha, Vargas, \& Davila, 2004; Munte, Serradell, \& Sorde, 2011).

El Plan integral del Pueblo Gitano en Catalunya asegura esta participación activa e informada de la comunidad gitana desde diferentes espacios. Un primer espacio sería el Consejo Asesor del Pueblo Gitano, órgano colegiado desde el primer Plan (2005) adscrito al Departamento de Trabajo, Asuntos Sociales y Familias de la Generalitat de Catalunya encargado de validar, reformular y hacer seguimiento de todo aquello que acontece en relación a la implementación y desarrollo del Plan integral del Pueblo Gitano (Departament de Treball. Afers Socials i Famílies, 2018c). La presidencia de este órgano la ocupa el consejero/a del Departamento, la vicepresidencia corresponde al director general de Acción Cívica y Comunitaria, y la vicepresidencia segunda la ejerce una persona gitana nombrada por la consejera a petición de las entidades representantes del Consejo Asesor. Este órgano está compuesto por 21 vocalías ocupadas por personas del pueblo gitano representantes de asociaciones y fundaciones, así como por personas gitanas que participan a título personal. La Generalitat de Catalunya rinde cuentas a este Consejo Asesor sobre cada una de las acciones que, bajo el Plan Integral, los Departamentos llevan a 
cabo, así como los resultados obtenidos, el presupuesto destinado (al céntimo), y el proceso de renovación del Plan Integral en su conjunto (García-Espinel, 2016).

Más allá del Consejo Asesor, podemos afirmar que la participación e implicación de la población gitana en el Plan Integral ha sufrido un importante salto cualitativo desde el tercer Plan (2014-2016), y muy especialmente desde el cuarto Plan (2017-2020).

Esta implicación se observa por ejemplo en los grupos de trabajo. Con el fin de hacer más operativa la implementación de las actuaciones previstas en los diferentes ámbitos del Plan, estos grupos de trabajo están compuestos por personas gitanas con experiencia en el ámbito concreto del grupo de trabajo, conjuntamente con técnicos/as y/o cargos del Departamento(s) vinculado al ámbito en cuestión (Departament de Treball. Afers Socials i Famílies, 2018c). Estos grupos de trabajo asesorar, temporizan, hacen el seguimiento y evalúan la implementación de acciones concretas del Plan, convirtiéndose en la mayoría de ocasiones en un puente de comunicación real, efectivo y rápido entre el Plan y la comunidad gitana. Algunos de estos grupos de trabajo, creados a partir del tercer Plan (2009) han cubierto los ámbitos de trabajo, salud, vivienda, educación, mujer, cultura o juventud. En este sentido, el cuarto Plan (2017-2020) prevé la creación de nuevos grupos de trabajo, como uno sobre Antigitanismo, con el fin de realizar recomendaciones y proponer acciones concretas para luchar contra esta forma específica de racismo persistente, sistemática y frecuentemente acompañada de episodios de violencia que sufre la comunidad gitana (ECRI, 2011).

Sin embargo, la implicación de la población gitana en el Plan Integral, concretamente en el diseño, el desarrollo, la implementación y el seguimiento del mismo, ha cambiado de un modo radical gracias a tres espacios de participación: 1) el equipo de renovación, 2) el equipo técnico multicultural, y 3) la contratación de personas gitanas para la implementación y desarrollo de las acciones del Plan.

Al igual que en el tercer Plan integral (2009-2013), en la elaboración del actual cuarto Plan (2017-2020) se ha creado un equipo de renovación del Plan integral del pueblo gitano, compuesto por un total de veinte personas (nueve mujeres gitanas, ocho hombres gitanos, dos mujeres no gitanas y un 
hombre no gitano). Este grupo, compuesto por un $85 \%$ de personas gitanas representantes de asociaciones y entidades, así como personas gitanas no asociadas, se ha encargado de definir todas y cada una de las acciones que se incluyen en el Plan Integral. En total, el equipo de renovación se ha reunido un total de ocho sesiones (todas ellas en 2016) para diseñar, enmendar y validar las medidas del Plan, así como para hacer otras aportaciones relacionadas con el Plan (Departament de Treball. Afers Socials i Famílies, 2018c). Este equipo de renovación ha tenido en cuenta también las aportaciones que han surgido del proceso participativo del cuarto Plan, el cual ha consistido en la realización de sesiones de debate en diferentes localidades que tienen una tasa elevada de población gitana, como son Reus, Viladecans, Barcelona y Terrassa. Estas sesiones han permitido recoger las aportaciones de la comunidad gitana durante el proceso de diseño y de desarrollo del Plan, especialmente de las personas gitanas que tradicionalmente no participan en la elaboración de políticas públicas (Departament de Treball. Afers Socials i Famílies, 2018c). Este proceso participativo también se promovió a través de un espacio abierto en línea en la página web de la Generalitat de Catalunya, con el fin de recibir otras aportaciones sobre los cuatro ejes principales que estructuran el cuarto Plan integral: educación, trabajo, vivienda y salud.

Otro espacio que demuestra la implicación de la población gitana en el Plan Integral es el nuevo equipo técnico multicultural que desarrolla e implementa las acciones del Plan desde el Departamento de Trabajo, Asuntos Sociales y Familias. El cuarto Plan ha supuesto un hecho histórico en la composición de este nuevo equipo multicultural, incorporando personas gitanas profesionales al equipo técnico del Programa del Pueblo Gitano y la Innovación Social; programa bajo el que se enmarca el Pla Integral. Este equipo está formado por 6 profesionales gitanos y 3 no gitanos, lo que supone que el $67 \%$ (es decir, 2/3) del equipo técnico que implementa y desarrolla el Plan Integral desde el Departamento competente, está formado por personas gitanas (Macías-Aranda, GarcíaEspinel, Álvarez-Jiménez, \& Cabré-Olivé, 2018).

Finalmente, podemos afirmar que el Plan integral garantiza la implicación activa e informada de la población gitana gracias a la contratación de personas gitanas, que son quienes implementan y 
desarrollan las acciones del Plan en todo el territorio catalán. Gracias a esta apuesta de la Generalitat de Catalunya, más de 50 personas gitanas han sido ya contratadas como docentes, gestores económicos, directores de equipamientos públicos, dinamizadores, mediadores u otros profesionales técnicos y superiores (Macías-Aranda, García-Espinel, et al., 2018).

En segundo lugar, otro de los principios clave del Plan Integral hace referencia a la consonancia de las medidas y acciones del Plan con los resultados de las investigaciones científicas de mayor nivel e impacto social. Tanto la comunidad gitana, como los principales organismos internaciones, así como la comunidad científica en su conjunto, vienen denunciando desde hace décadas la ineficacia y consecuencias de la implementación y desarrollo de investigaciones e intervenciones que no están basadas en las recomendaciones científicas, contribuyendo así a reproducir las situaciones de desigualdad de la comunidad gitana (Amador, 2016; ECRI, 2011; Flecha, 2004; Hancock, 1988, 2000; Macías-Aranda \& Redondo, 2012; McGarry, 2011; Sordé-Martí \& Macías-Aranda, 2017; Sordé, Flecha, \& Mircea, 2013).

Con el fin de superar esta visión reproduccioncita y conseguir mejorar de un modo real y efectivo la situación de la comunidad gitana en Catalunya, el Plan Integral basa sus medidas y acciones en los resultados y recomendaciones de las investigaciones científicas de máximo nivel internacional y mayor impacto social. Si observamos el actual cuarto Plan, existe un apartado específico e íntegramente dedicado a la recopilación de evidencias científicas y otras experiencias que están consiguiendo alcanzar la inclusión de los grupos más vulnerables, y particularmente del pueblo gitano (Departament de Treball. Afers Socials i Famílies, 2018c).

Entre las investigaciones recogidas por el Plan, destaca en el ámbito educativo el proyecto INCLUD-ED "Estrategias para la inclusión y la cohesión social en Europa desde la educación" (2006-2011). Entre las numerosas contribuciones científicas del proyecto INCLUD-ED, destacan las Actuaciones Educativas de Éxito, ${ }^{3}$ un conjunto de actuaciones que están obteniendo los mejores resultados académicos y de convivencia en todos los contextos en los que se aplican (Flecha, 2015). Estas Actuaciones Educativas de Éxito se han demostrado especialmente beneficiosas para los grupos más vulnerables, como el caso del Pueblo Gitano, contribuyendo de 
un modo claro a la reducción del absentismo escolar, la mejora de la convivencia, el aumento de la matrícula escolar, la mejora de la relación familia - centro educativo, la reducción del fracaso escolar, la mejora del rendimiento académico del alumnado, la mejora de la formación educativa de las familias, el aumento de las expectativas educativas y sociales de toda la comunidad educativa, y/o la transformación de las desigualdades sociales y educativas del entorno (Flecha \& Soler, 2013; Girbés-Peco, MacíasAranda, \& Álvarez-Cifuentes, 2015; Macías-Aranda, 2017; Sordé-Martí \& Macías-Aranda, 2017; Valls \& Kyriakides, 2013). De hecho, dado el impacto de las Actuaciones Educativas de Éxito, así como el impacto alcanzado por otros resultados del proyecto, INCLUD-ED ha sido el único proyecto de ciencias sociales y humanidades destacado por la Unión Europea en su lista de las 10 investigaciones de éxito del Programa Marco de Investigación (European Commission, 2011b), que es el programa más prestigioso y de mayor dotación de recursos del mundo.

Todavía en el ámbito educativo, el Plan integral basa algunas de sus acciones en los resultados de otras investigaciones, como las desarrolladas por el Proyecto de Derechos Civiles (CRP) sobre los efectos y beneficios de las políticas de acción afirmativa (Orfield, 2001a, 2001b, 2009). Esta línea de investigación fue iniciada por el CRP en la prestigiosa Universidad de Harvard, y se sigue desarrollando en la actualidad en la Universidad de California - Los Ángeles (UCLA).

Por lo que respecta a otras áreas cruciales, el Plan Integral también tiene en cuenta las recomendaciones y resultados de otros trabajos científicos, tales como: la aportación de la profesora de la Facultad de Salud Pública de la Universidad de Harvard, Rima Rudd, en relación al concepto y los beneficios de la alfabetización en salud; el concepto de Vivienda Social y sus beneficios, desarrollado por Vaillancourt et al. (2001) en la Universidad de Quebec y Montreal a partir de una experiencia de éxito; o el concepto de alfabetización financiera y sus beneficios en múltiples áreas de la vida, desarrollado nuevamente por la Universidad de Harvard gracias al programa "Joint Center for Housing Studies".

Los últimos aspectos clave del Plan Integral hacen referencia a la colaboración y diálogo permanente con las autoridades locales y la administración pública., así como a la existencia de indicadores concretos, 
realistas y cuantificables de impacto, que tienen como objetivo primero y último mejorar la situación social de esta comunidad.

En relación a la colaboración y diálogo permanente con las autoridades locales y la administración pública, el Plan integral cuenta desde su inicio con una Comisión Interdepartamental donde están representados todos los departamentos de la Generalitat de Catalunya que deben implementar y/o desarrollar acciones y o medidas específicas en favor de la comunidad gitana. En esta comisión el Plan Integral consigue que los diferentes departamentos se comprometan a dar cumplimiento a las acciones del Plan que les afectan, así como a destinar una partida presupuestaria concreta para dichas actividades (Departament de Treball. Afers Socials i Famílies, 2018c; García-Espinel, 2016). Además de este espacio de participación, en el que también están representados diferentes miembros gitanos del Consejo Asesor, la comunicación entre el equipo técnico del Plan Integral y el resto de equipos técnicos de la Generalitat de Catalunya es fundamental para el desarrollo y cumplimiento de las medidas del Plan, ya que muchas de estas acciones dependen mayoritariamente del trabajo, la voluntad y la eficacia de otras unidades diferentes a la del Plan Integral. Así mismo, los convenios de colaboración que el Plan Integral mantiene con otras administraciones publicas de Catalunya, como ayuntamientos o consejos comarcales, garantizan la implementación y desarrollo de un modo eficaz de las políticas del plan en todo el territorio catalán (Departament de Treball. Afers Socials i Famílies, 2018c; García-Espinel, 2016).

Finalmente, por lo que respecta a la existencia de indicadores concretos, realistas y cuantificables de impacto, desde la tercera edición del Plan Integral (2014-2016), las acciones y medidas del Plan están vinculadas a indicadores de impacto que permiten conocer la eficiencia de las actuaciones y la consecución de los resultados esperados (Departament de Benestar i Família, 2014; Departament de Treball. Afers Socials i Famílies, 2018c). Estos indicadores, recomendados por las organizaciones más relevantes dentro del ámbito (EU-FRA, 2009; European Commission, 2015, 2016), permiten saber si efectivamente las acciones del Plan están contribuyendo o no a transformar la realidad actual y favorecer la inclusión del pueblo gitano en Cataluña. Este aspecto, aumenta no únicamente la eficacia y transparencia del Plan, si no que permite reorientar las acciones 
del mismo con el objetivo último de alcanzar una mejora social cuantificable en la comunidad gitana (García-Espinel, 2016).

\section{Impacto social del Plan Integral del Pueblo Gitano en Catalunya}

Los principios clave del Plan Integral del Pueblo Gitano en Catalunya señalados en el apartado anterior explican el impacto social que se está alcanzando en la actualidad en Catalunya con respecto a la comunidad gitana. A continuación, se presentan y discuten algunos de estos impactos. En primer lugar, explicamos el impacto en la educación primaria. Seguidamente, explicamos el impacto en el acceso a la educación y al mercado laboral de las personas gitanas adultas.

\section{Avances en educación primaria}

Si nos centramos en el ámbito educativo, el Plan integral está promoviendo la implementación de Actuaciones Educativas de Éxito en 7 escuelas de atención prioritaria. Estas escuelas, situadas en todo el territorio catalán, tienen una alta proporción de alumnado gitano y se encuentran en entornos extremadamente vulnerables. Una de estas escuelas está situada en uno de los barrios más pobres de la provincia de Tarragona, con índices elevadísimos de desempleo, analfabetismo y privación de recursos. Concretamente, la composición del alumnado de esta escuela es de más del $65 \%$ de origen gitano, albergando también a más del $25 \%$ de procedencia marroquí, y el resto (aproximadamente un 6\%) de otras procedencias, mayoritariamente de origen inmigrante, como Rumania o India, entre otros.

La implementación de Actuaciones Educativas de Éxito en esta escuela desde 2012 (implementación promovida muy especialmente por el Plan Integral desde 2014), ha conseguido, por ejemplo, reducir el absentismo drásticamente. En 2011, antes de la implementación de estas acciones, el absentismo se situaba por encima del $46 \%$. En 2015, tres cursos después, este dato era del $0,94 \%$, lo que nos permite afirmar que el absentismo prácticamente ha desaparecido en esta escuela (véase Gráfico 1). 


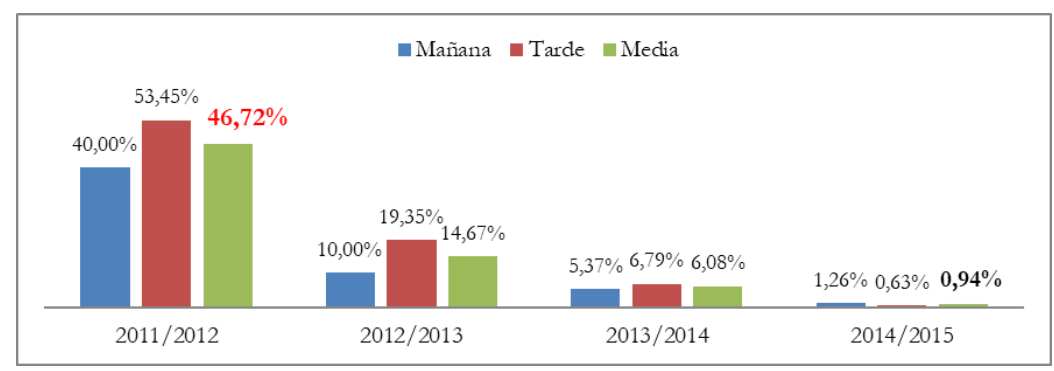

Figura 1. Evolución del Absentismo (mañana, tarde y media global) (2011/2012 2014/2015). Centro de Atención Prioritaria del Plan Integral del Pueblo Gitano de Catalunya (Macías-Aranda, 2017, p. 164).

Esta reducción del absentismo ha venido acompañada de una notable mejora del nivel de aprendizaje del alumnado. Esta mejora se observa espacialmente en etapas como la de educación infantil. Tal y como se puede observar en el Gráfico 2, en el curso 2011-2012, el porcentaje de alumnado de P5 que reconocía sonidos silábicos no alcanzaba el 32\%. Sin embargo, en el curso 2014-2015, es decir, tres cursos después de implementar Actuaciones Educativas de Éxito, el porcentaje de los alumnos de P3 (alumnado entre 1 y 2 años menor que el alumnado de P5) que leía y/o escribía sonidos silábicos superaba el $92 \%$.

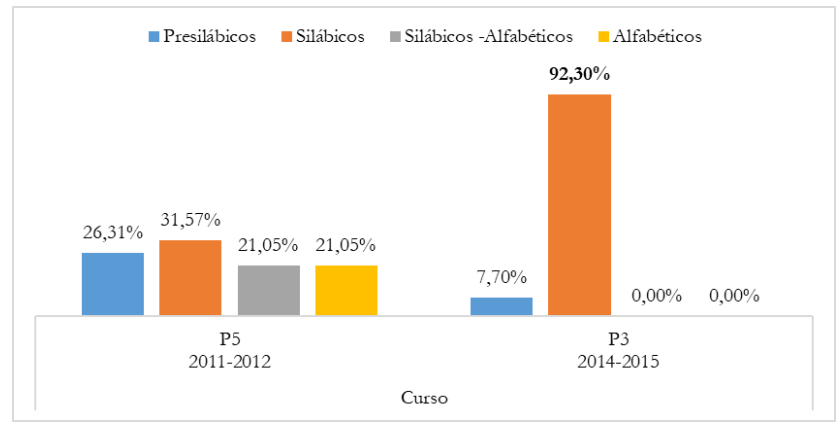

Figura 2. Lectoescritura en Educación Infantil (P3-P5) (2011-2012 y 2014-2015). Centro de Atención Prioritaria del Plan Integral del Pueblo Gitano de Catalunya (Macías-Aranda, 2017, p. 161). 
En lo que se refiere a la etapa de la educación primaria (alumnado de entre 6 y 12 años), las mejoras en este centro son también claramente notables. Por ejemplo, en tan solo un curso académico implementando Actuaciones Educativas de Éxito (entre 2011-2012 y 2012-2013), el porcentaje de alumnado que superó exitosamente los objetivos de $4^{\circ}$ de primaria pasó del 30\% al 60\% (Macías-Aranda, 2017, p. 161). Si atendemos a los resultados de las pruebas diagnósticas oficiales de competencias básicas realizadas por el Departamento de Educación de la Generalitat de Catalunya, las mejoras alcanzadas son claramente evidentes. Tal y como se observa en el gráfico 3 , el alunado de tercero de primaria que ha superado con éxito dichas pruebas ha pasado del 18,18\% en el curso 2015-2016 al 91,66\% en el curso 2017-2018 (Departament de Treball. Afers Socials i Famílies, 2018a).

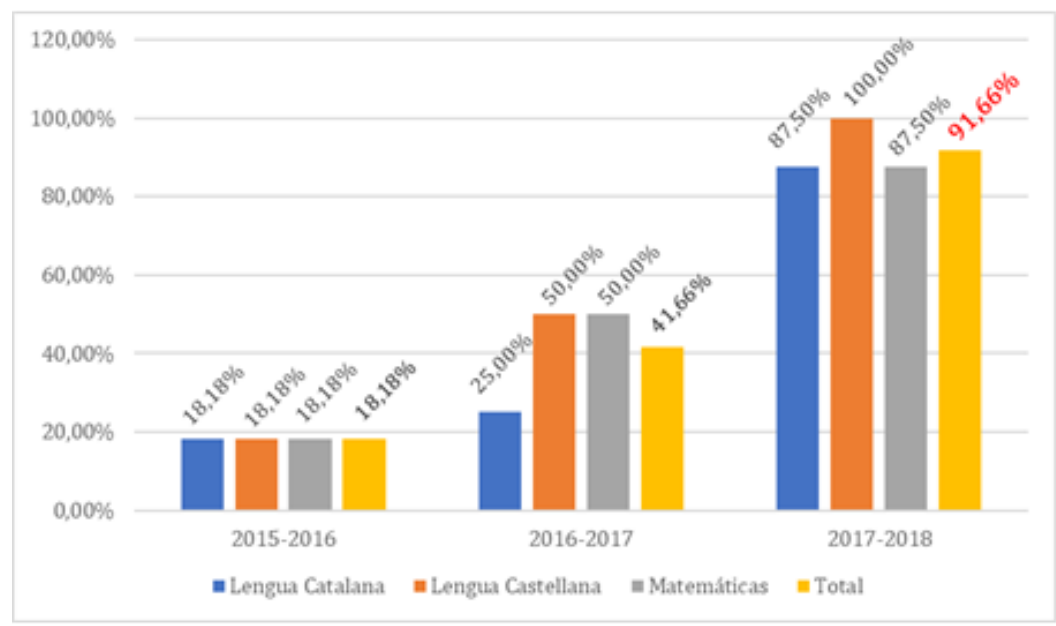

Figura 3. Pruebas diagnósticas de Competencias Básicas (2015-2016 - 2017-2018). Centro de Atención Prioritaria del Plan Integral del Pueblo Gitano de Catalunya (Departament de Treball. Afers Socials i Famílies, 2018a).

Desafortunadamente, los logros alcanzados por este centro todavía no se están manifestando con tanta notoriedad en el resto de centro de atención prioritaria del Plan Integral. Los motivos que explican dichas diferencias 
hacen referencia al tiempo que los centros llevan implementando estas Actuaciones Educativas de Éxito (el resto de centros recién han empezado a implementarlas en 2016-2017) y a la implementación parcial de estas acciones (únicamente el centro de la provincia de Tarragona implementa todas las Actuaciones Educativas de Éxito, mientras que el resto de centros de atención prioritaria únicamente implementa algunas de estas acciones, como los grupos Interactivos, las tertulias dialógicas o la formación de familiares) (Departament de Treball. Afers Socials i Famílies, 2018a).

\section{Avances en educación superior y acceso al mercado laboral}

Otro de los impactos alcanzados por el Plan Integral hace referencia a la mejora de la situación educativa y laboral de la población gitana adulta. Desde el año 2012, y siguiendo los principios de la formación de familiares, una de las Actuaciones Educativas de Éxito, se está desarrollando un curso de preparación para superar las pruebas oficiales de acceso a la universidad para mayores de 25 años, dirigido a personas gitanas. Esta acción se alinea con uno de los objetivos específicos del Plan Integral desde 2014: incrementar el número de personas gitanas que cursan estudios postobligatorios, y concretamente en estudios universitarios (Departament de Treball. Afers Socials i Famílies, 2018c).

La asistencia de personas gitanas a este curso ha aumentado progresivamente durante sus cinco ediciones. Desde las 6 personas gitanas matriculadas (15 inscritas) en la primera edición (2011-2012), a 24 personas matriculadas (55 inscritas) en la última edición de 2017-2018 (Departament de Treball. Afers Socials i Famílies, 2018b; Macías-Aranda, 2017). Las personas matriculadas son las personas gitanas que después de asistir al curso se matriculan para presentarse a la prueba oficial. Las personas inscritas son personas gitanas que inician el curso, pero por diferentes motivos deciden no matricularse a la prueba oficial de ese año (Macías-Aranda, 2017).

Este aumento de la asistencia de estudiantes se explica gracias al éxito del curso, ya que poco a poco, el número de personas gitanas que han accedió a la universidad ha ido aumentando considerablemente. Hasta la sexta edición 2017-2018, un total de 19 gitanas y gitanos han superado la 
prueba oficial de acceso a la universidad para mayores de 25 o 45 años, las cuales se encuentran cursando grados universitarios en las principales universidades de Catalunya, como los grados de Sociología, Educación, Psicología, Economía, Trabajo Social, Educación Social, Medicina o Enfermería, entre otros (Departament de Treball. Afers Socials i Famílies, 2018b; Macías-Aranda, 2017).

Es importante señalar que, en la última prueba oficial de 2018, además de las que superaron el examen en su totalidad, 9 personas gitanas aprobaron la mitad del examen, teniendo que superar en 2019 una única parte para acceder a la universidad (Departament de Treball. Afers Socials i Famílies, 2018b).

El éxito de estas personas gitanas se ha transferido a todo su entorno familiar y social, aumentando las expectativas sociales, y especialmente educativas de sus hijas e hijos. Javier, por ejemplo, es un padre gitano que aprobó la prueba en 2017 y actualmente estudia el grado de Trabajo social en una universidad pública catalana. Javier se ha criado y actualmente sigue residiendo en uno de los barrios más desfavorecidos de la provincia de Barcelona, donde conviven una alta proporción de población gitana. Gracias a que él se encuentra estudiando en la universidad, las expectativas educativas de sus hijas se han transformado totalmente, tal y como él nos lo explica:

Desde que yo voy a la universidad mi vida ha cambiado totalmente. Las niñas por ejemplo ahora me preguntan por los exámenes, y se ponen muy contentas por mí cuando les digo que he parobado algo. (...) mi grande dice que quiere ser como yo, y que cuando sea grande quiere estudiar en la Universidad y Trabajo Social como su papa (Departament de Treball. Afers Socials i Famílies, 2018b)

Por su parte, Rocío es una madre gitana de dos niños que ha crecido (y sigue residiendo) en uno de los barrios más desfavorecidos de Catalunya, donde residen un gran número de familias gitanas. Rocío aprobó la prueba de acceso en 2018 y actualmente se encuentra estudiando Medicina en una universidad pública catalana. El impacto personal, familiar y social de su acceso a la universidad ha sido muy claro, tal y como ella misma nos explica: 
Yo todavía no me lo creo, la verdad. Estoy muy contenta y muy ilusionado. No está siendo fácil, pero estoy haciendo lo que siempre quise hacer, y eso no tiene precio. (...) ahora incluso las profesoras o las asistentas sociales me tratan diferente cuando les digo que estudio medicina (...), he podido trabajar en varias cosillas así del mundo asociativo, pero sobre todo el cambio lo veo en la casa. Mi madre, mi padre, los niños... Ahora en la casa no se habla de otra cosa que no sea la Universidad (risas) (Departament de Treball. Afers Socials i Famílies, 2018b)

Otro impacto alcanzado en relación a las personas gitanas que han accedido a la universidad gracias a esta formación de familiares del Plan Integral, hace referencia a la mejora de sus condiciones de vida, y concretamente laborales. El $88 \%$ de las personas gitanas que han superado el examen han mejorado sus condiciones laborales, gracias a incrementar sus niveles educativos, y así su empleabilidad. Esta mejora ha supuesto para muchas familias gitanas dejar atrás trabajos muy precarios y de gran desgaste físico, como el mercadillo o la recolección de chatarra (MacíasAranda, 2017).

Moisés es un padre gitano de 4 hijas e hijos que superó el examen en 2017 y que actualmente se encuentra estudiando el grado de sociología en una universidad pública catalana. Durante años, Moisés se dedicó a la búsqueda y recolección de chatarra, y gracias a superar la prueba, en 2017 empezó a trabajar como dinamizador sociocultural en un ayuntamiento de Catalunya, y actualmente se encuentra trabajando como técnico socioeducativo para la administración pública. Este importante cambio laboral ha supuesto una mejora en las condiciones de vida de Moisés y de toda su familia, tal y como nos lo explica a continuación:

El cambio en mi vida ha sido muy grande. Yo antes no sabía cuánto dinero iba a sacarme con la chatarra, y ahora cada mes tienes una nómina. (...) En casa somos muchos, y claro que vas justo, pero no tiene nada que ver con antes. Ahora, por ejemplo, el mero hecho de tener una nómina me ha permitido poder pedir dos préstamos personales para arreglarme la casa y para comprar un coche. Eso antes era imposible. (...) Eso por no hablar del cambio en la 
mentalidad de mis hijas que ahora me hablan de la universidad como si nada, o en las condiciones laborales, que aunque me paso muchísimas horas en el coche, trabajo en algo que me gusta y sin pasar calamidades por el frio o la calor (Departament de Treball. Afers Socials i Famílies, 2018b)

Este éxito social y educativo ha abierto camino a nuevos escenarios de gran importancia para el acceso igualitario de la población gitana a los estudios superiores. Uno de estos escenarios llega desde la propia sociedad civil gitana, con la creación de la primera red gitana universitaria de Catalunya, CampusRom. Esta red solidaria de apoyo mutuo entre personas gitanas que intentan acceder, o ya lo han conseguido, a estudios postobligatorios está generando nuevos referentes para la comunidad gitana, y el impacto de la red ya ha sido evidenciado. La red CampusRom, que surge en el marco del curso de acceso del Plan Integral y que está estrechamente ligada a él, aunque es totalmente independiente, consiguió en 2017 que, por primera vez en la historia del curso, 7 personas gitanas accedieran en una misma edición. Además, CampusRom ayudó a otras personas gitanas a acceder a la universidad por otras vías, lo que supuso que, en 2017, 13 personas gitanas accedieran a estudios posobligatorios (Macías-Aranda, García-Carrión, Valls, \& Girbés, 2018). La red cuenta ya con más de 60 personas gitanas que están cursando estudios postobligatorios o que están intentando acceder a la educación superior, y en 2018 ha creado una delegación en Aragón.

\section{Conclusiones}

El marco político en favor de la comunidad gitana ha sufrido un cambio muy importante en los últimos 10 años, pero pese a que nos encontramos ante el contexto político más favorable de la historia en el trabajo para la mejora de la situación del Pueblo Gitano, cabe tener en cuenta que dicho marco propicia, pero no garantiza los resultados de éxito si, al mismo tiempo, no incluimos en nuestra intervención algunos elementos clave que se desarrollan en el presente artículo.

Los modelos y espacios de participación gitana que despliega el Plan integral, el trabajo a partir de evidencias científicas, la creación de equipos 
interculturales investigadores y/o técnicos, la orientación a resultados de impacto y responsabilidad -política y/o científica- con la transformación social de la población a la que nos dirigimos, configuran una arquitectura sólida para propiciar la equiparación socioeconómica y cultural del Pueblo Gitano con el resto de la sociedad de la cual forman parte.

Este artículo pone de manifiesto el significativo impacto social que se alcanza cuando se incorporan en los planes específicos en favor de la comunidad gitana los elementos clave que se desarrollan en el Plan Integral del Pueblo Gitano en Catalunya. Mejoras en los niveles de aprendizaje y reducción del absentismo escolar del alumnado gitano, transformación de las expectativas sociales y educativas de la comunidad gitana en su conjunto, equiparación de la situación socioeducativa de las familias gitanas a través de su acceso a la universidad o al mercado laboral cualificado, son algunos de los impactos evidenciados por las investigaciones científicas en las que se basa este artículo.

A su vez, se ha demostrado en este artículo cómo este impacto social puede trascender de los propios planes gitanos, facilitando la aparición de nuevos movimientos sociales que luchan por el éxito de esta comunidad (como sería el caso de CampusRom, la primera red gitana universitaria de Catalunya) o alentando a otras administraciones a desarrollar medidas específicas en favor de la comunidad gitana (como sería el caso de la reserva de plazas universitarias para personas gitanas promovida por la Secretaria de Universidades de la Generalitat de Catalunya a través del Consejo Interuniversitario de Catalunya).

Todos los elementos clave desarrollados en el artículo son transferibles a cualquier contexto y pueden incorporarse desde la intervención social y desde el ámbito académico, científico y político. Esta transferibilidad es de vital importancia para aumentar las probabilidades de éxito de la comunidad gitana más allá de Catalunya.

Por último, cabe destacar la contribución que tratamos de realizar en la mejora de la imagen social del Pueblo Gitano y en la superación el Antigitanismo. La mejora de los resultados educativos de la población gitana y su participación igualitaria en espacios de decisión contribuye a aumentar el diálogo intercultural y la superación de estereotipos sociales inherentes a esta comunidad. Consideramos especialmente relevante esta 


\section{García-Espinel, et al. - Plan Integral Pueblo Gitano Catalunya}

contribución en el momento actual, con un creciente espacio político ocupado por formaciones de extrema derecha en Europa. Las políticas y proyectos que contribuyen a mejorar la situación e imagen social de la minoría más numerosa del continente europeo, inciden, tal y como se evidencia en el artículo, en una mayor cohesión y convivencia europea. La participación decisoria del Pueblo Gitano en las políticas que le afectan contribuye a incentivar un concepto de ciudadanía gitana menos vulnerable a corrientes xenófobas y racistas, con mayores oportunidades de defender sus derechos en tanto que ciudadanos y en tanto que representantes de la minoría cultural más importante de nuestro continente.

\section{Notas}

1 Las Actuaciones Educativas de Éxito se pueden encontrar explicadas con detalle en numerosas publicaciones científicas, por ejemplo en Flecha (2015), así como en el propio documento oficial del IV Plan Integral del Pueblo Gitano en Catalunya (Departament de Treball. Afers Socials i Famílies, 2018c), o en por el Departamento de Educación de la Generalitat de Catalunya (XTEC - Xarxa Telemàtica Educativa de Catalunya, 2018). Estas actuaciones son las siguientes: 1) los grupos interactivos, 2) las tertulias dialógicas, 3) la formación de familiares, 4) las actuaciones de extensión del tiempo de aprendizaje, como la biblioteca tutorizada, 5) la participación educativa de la comunidad, y 6) el modelo dialógico de prevención y resolución de conflictos

\section{Bibliografía}

Aiello, E., Mondejar, E., \& Pulido, M. Á. (2013). Communicative Methodology of Research and Recognition of the Roma People. International Review of Qualitative Research, 6(2), 254-265. doi: 10.1525/irqr.2013.6.2.254

Amador, J. (2016). La "Roma response" al modelo reproduccionista. La educación, nuestra escalera para la transformación social.

International Journal of Sociology of Education, 5(2), 144-163. doi: 10.17583/rise.2016.2091

Brüggemann, C., \& Friedman, E. (2017). The decade of Roma inclusion: Origins, actors, and legacies. European Education, 49(1), 1-9. doi: $10.1080 / 10564934.2017 .1290422$ 
Comisión Europea. (2009). Vademécum. Los 10 Principios básicos comunes para la inclusión de los gitanos.

Congreso de los Diputados (2005) Proposición no de Ley relativa al reconocimiento de los derechos del pueblo gitano, 162/000320.

BOCG., serie D, número 186. Madrid, 15 de abril de 2005

Consejo de la Unión Europea (2013). Recomendación relativa a la adopción de medidas eficaces de integración de los gitanos en los Estados miembros. (2013/C 378/01). Luxemburg, 9.12.2013. Retrieved from http://eur-lex.europa.eu/legalcontent/ES/TXT/PDF/?uri=CELEX:32013H1224(01)\&from=ES

Council of Europe (2012a). Declaration Decl-01.02.2012E of the Committee of Ministers on the Rise of Anti-Gypsyism and Racist Violence against Roma in Europe. Strasbourg.

Council of Europe (2012b). Descriptive Glossary of terms relating to Roma issues, (May), 28.

Damonti, P., \& Arza, J. (2014). Exclusión en la comunidad gitana: Una brecha social que persiste y se agrava. Madrid: Fundación Foessa. Retrieved from http://www.foessa2014.es/informe/uploaded/documentos_trabajo/15 102014151523_8331.pdf

Departament de Benestar i Família. Generalitat de Catalunya. (2005). Estudi sobre la població gitana a Catalunya. Barcelona: Generalitat de Catalunya. Retrieved from

http://treballiaferssocials.gencat.cat/web/.content/01departament/08p ublicacions/ambits_tematics/serveis_socials/08estudipoblaciogitanac at05/2005estudipoblaciogitanacat.pdf

Departament de Benestar i Família. (2014). Pla integral del poble gitano de Catalunya 2014-2016. Barcelona: Generalitat de Catalunya.

Retrieved from

http://treballiaferssocials.gencat.cat/web/.content/03ambits_tematics/ 18_accio_comunitaria_i_voluntariat/01_accio_comunitaria/04_pla_i ntegral_poble_gitano/pipg2014.pdf

Departament de Treball. Afers Socials i Famílies. (2018a). Informe de seguimiento de los Centros de Atención Prioritaria del Plan Integral del Pueblo Gitano en Catalunya. Barcelona. 
Departament de Treball. Afers Socials i Famílies. (2018b). Informe de seguimiento del GAU25. Barcelona.

Departament de Treball. Afers Socials i Famílies. (2018c). Plan Integral del Pueblo Gitano en Catalunya (2017-2020).

ECRI. European Commission against Racism and Intolerance, \& Council of Europe. General Policy CRI(2011)37 Recommendation No. 13 on combating anti-Gypsyism and discrimination against Roma (2011). Strasbourg.

EU-FRA. European Union Agency for Fundamental Rights. (2009). Selected positive initiatives. The situation of Roma EU citizens moving to and settling in other EU Member States. Luxembourg: Offi ce for Offi cial Publications of the European Communities. doi: $10.2811 / 34658$

EU-FRA. European Union Agency for Fundamental Rights. (2012). The situation of Roma in 11 EU Member States. Luxembourg: Publications Office of the European Union. Retrieved from http://fra.europa.eu/sites/default/files/fra_uploads/2099-FRA-2012Roma-at-a-glance_EN.pdf

EU-FRA. European Union Agency for Fundamental Rights. (2014a). Roma survey - Data in focus Discrimination against and living conditions of Roma women in 11 EU Member States. Luxembourg: European Union Agency for Fundamental Rights. doi: 10.2811/52654

EU-FRA. European Union Agency for Fundamental Rights. (2014b). Roma survey - Data in focus Education: the situation of Roma in $11 \mathrm{EU}$ Member States. Luxembourg: Publications Office of the European Union. Retrieved from http://fra.europa.eu/sites/default/files/fra2014_roma-survey_education_tk0113748enc.pdf

EU-FRA. European Union Agency for Fundamental Rights. (2014c). Roma survey - Data in focus Poverty and employment: the situation of Roma in 11 EU Member States. Vienna: European Union Agency for Fundamental Rights. doi: 10.2811/50875

EU-FRA. European Union Agency for Fundamental Rights. (2018). A persisting concern: anti-Gypsyism as a barrier to Roma inclusion. doi: $10.2811 / 423901$ 
European Commission (2011a). Communication from the Commission to the European Parliament, The Council, The European Economic and Social Committee and the Committee of the Regions, An EU

Framework for National Roma Integration Strategies up to 2020,

$\operatorname{COM}(2011) 173$ final. Brussels, 5 April 2011

European Commission. (2011b). Added Value of Research, Innovation and

Science portfolio. Brussels. Retrieved from

http://europa.eu/rapid/press-release_MEMO-11-520_en.pdf

European Commission. (2011c). An EU Framework for National Roma

Integration Strategies up to 2020. COM(2011) 173 final. Brussels,

5.4.2011. Retrieved from

http://ec.europa.eu/health/social_determinants/docs/com2011_173_e n.pdf

European Commission. (2015). Report on the implementation of the EU

Framework for National Roma Integration Strategies 2015. Brussels,

17.6.2015 COM(2015) 299 final. Retrieved from

http://ec.europa.eu/justice/discrimination/files/roma_communication

2015_en.pdf

European Commission. (2016). Assessing the implementation of the EU

Framework for National Roma Integration Strategies and the

Council Recommendation on effective Roma integration measures in the Member States - 2016. Brussels, 27.6.2016 COM (2016) 424.

Retrieved from http://ec.europa.eu/justice/discrimination/files/romareport-2016_en.pdf

European Parliament (2008) European Parliament resolution on a European

Strategy on the Roma, P6_TA(2008)0035. Brussels, 28 January 2008 European Parliament. (2015). Resolution on the occasion of International

Roma Day - anti-Gypsyism in Europe and EU recognition of the memorial day of the Roma genocide during World War II $(2015 / 2615(R S P))$. Brussels. Retrieved from http://www.europarl.europa.eu/sides/getDoc.do?pubRef=//EP//NONSGML+TA+P8-TA-2015-0095+0+DOC+PDF+V0//EN

European Parliament (2005). European Parliament resolution on the situation of the Roma in the European Union, P6_TA(2005)0151. Brussels, 28 April 2005. Retrieved from: 
115 García-Espinel, et al. - Plan Integral Pueblo Gitano Catalunya

http://www.europarl.europa.eu/sides/getDoc.do?pubRef=//EP//TEXT+TA+P6-TA-2005-0151+0+DOC+XML+V0//EN

Fernández, M., Domínguez, C., \& Flecha, A. (2004). Mujeres gitanas y mercado laboral: mecanismos para superar su triple exclusión. Lan Harremanak: Revista de Relaciones Laborales, 11, 81-94. Retrieved from

https://dialnet.unirioja.es/servlet/articulo?codigo=1067706\&info=res umen\&idioma=SPA

Flecha, R. (2004). Investigar desde la igualdad de las diferencias. In A. Touraine, M. Wieviorka, \& R. Flecha (Eds.), Conocimiento e identidad: Voces de grupos culturales en la investigación social (pp. 34-49). Barcelona: El Roure.

Flecha, R. (2006). INCLUD-ED. Strategies for inclusion and social cohesion from education in Europe. Brussels: INTEGRATED PROJECT. Priority 7 of Sixth Framework. European Commission.

Flecha, R. (2015). Successful Educational Actions for Inclusion and Social Cohesion in Europe. (R. Flecha, Ed.). Heidelberg \& New York \& Dordrecht \& London: Springer.

Flecha, R., \& Soler, M. (2013). Turning difficulties into possibilities: engaging Roma families and students in school through dialogic learning. Cambridge Journal of Education, 43(4), 451-465. doi. 10.1080/0305764X.2013.819068

Flecha, R., Vargas, J., \& Davila, A. (2004). Metodología comunicativa crítica en la investigación en ciencias sociales: la investigación WORKALÓ. LAN HARREMANAK, 2(11), 21-33.

García-Espinel, T. (2015). Contributions of Romani Women to Science. Policies and Social Improvement, 4(3), 832-855. doi:

10.4471/generos.2015.1721

García-Espinel, T. (2016). Impacto político de la investigación europea sobre el Pueblo Gitano. El caso del Plan Integral del Pueblo Gitano de Catalunya. Universidad de Granada.

Girbés-Peco, S., Macías-Aranda, F., \& Álvarez-Cifuentes, P. (2015). De la escuela gueto a una Comunidad de Aprendizaje: un estudio de caso sobre la superación de la pobreza a través de una educación de éxito. 
RIMCIS - International and Multidisciplinary Journal of Social Sciences, 4(1), 88-116. doi: 10.17583/rimcis.2015.04

Gómez, A., Puigvert, L., \& Flecha, R. (2011). Critical communicative methodology: Informing real social transformation through research. Qualitative Inquiry, 17(3), 235-245. doi:

10.1177/1077800410397802

Gómez, A., Racionero, S., \& Sordé, T. (2010). Ten years of critical communicative methodology. International Review of Qualitative Research, 3(1), 17-43.

Hancock, I. (1988). Reunification and the role of international Romani Union. Roma, (29), 9-19.

Hancock, I. (2000). The Consequences of Anti-Gypsy Racism in Europe. Other Voices, 2(1). Retrieved from http://www.othervoices.org/2.1/hancock/roma.php

Instituto Nacional de Estadística (INE). (2017). Población de 16 y más años por nivel de formación alcanzado, sexo y comunidad autónoma. Porcentajes resto del total. Madrid. Retrieved from http://www.ine.es/jaxiT3/Datos.htm?t=6369

Macías-Aranda, F. (2017). Contribuciones del Pueblo Gitano para luchar contra la Pobreza y el Antigitanismo a través de su participación en Actuaciones Educativas de Éxito. Universidad de Barcelona.

Macías-Aranda, F., \& Flecha, R. (2013). Hacia una formación del profesorado para la igualdad del Pueblo Gitano. Enseñantes Con Gitanos, (30), 75-84.

Macías-Aranda, F., García-Carrión, R., Valls, R., \& Girbés, S. (2018). CampusRom, the first Roma University Network of Catalonia. In ECER 2018. European Conference on Educational Research. Bolzano, Italia: EERA, European Educational Research Association. Macías-Aranda, F., García-Espinel, T., Álvarez-Jiménez, G., \& CabréOlivé, J. (2018). From The Ghetto To The University: The Social Impact Of The Integrated Plan For Roma People In Catalonia. Bolzano, Italia.

Macías-Aranda, F., \& Redondo, G. (2012). Pueblo gitano, género y educación : investigar para excluir o investigar para transformar. 
117 García-Espinel, et al. - Plan Integral Pueblo Gitano Catalunya

International Journal of Sociology of Education, 1(1), 71-92. doi: 10.4471/rise.2012.04

McGarry, M. (2011). The dilemma of the European Union's Roma policy.

Critical Social Policy, 32(1), 126-136. doi:

10.1177/0261018311425201

Ministerio de Sanidad. Política Social e Igualdad. (2011). Diagnóstico social de la comunidad gitana en España. Un análisis contrastado de la Encuesta del CIS a Hogares de Población Gitana 2007. Madrid: Ministerio de Sanidad, Política Social e Igualdad. Retrieved from https://www.msssi.gob.es/ssi/familiasInfancia/inclusionSocial/poblac ionGitana/docs/diagnosticosocial_autores.pdf

Ministerio de Sanidad. Servicios Sociales e Igualdad. (2012). Estrategia Nacional para la Inclusión Social de la Población Gitana en España 2012-2020. Madrid: Ministerio de Sanidad. Servicios Sociales e Igualdad. Retrieved from

https://www.msssi.gob.es/ssi/familiasInfancia/inclusionSocial/poblac ionGitana/docs/WEB_POBLACION_GITANA_2012.pdf

Ministerio de Sanidad. Servicios Sociales e Igualdad. (2013). Plan Operativo 2014-2016. Estrategia Nacional para la Inclusión Social de la Población Gitana 2012-2020. Madrid: Ministerio de Sanidad, Política Social e Igualdad. Gobierno de España. Retrieved from http://www.msssi.gob.es/ssi/familiasInfancia/inclusionSocial/poblaci onGitana/docs/PlanOperativoPoblacionGitana2014-2016.pdf

Ministerio de Sanidad. Servicios Sociales e Igualdad. (2016). Segunda Encuesta Nacional de Salud a Población Gitana 2014. Madrid: Ministerio de Sanidad, Política Social e Igualdad.

Ministerio de Sanidad. Servicios Sociales e Igualdad. (2018). Plan Operativo 2018-2020. Estrategia Nacional para la Inclusión Social de la Población Gitana 2012-2020.

Munte, a., Serradell, O., \& Sorde, T. (2011). From Research to Policy: Roma Participation Through Communicative Organization.

Qualitative Inquiry, 17(3), 256-266. doi:

$10.1177 / 1077800410397804$ 
Orfield, G. (2001a). Diversity challenged: Evidence on the impact of affirmative action. (Harvard Civil Rights Project \& Harvard Education Publishing Group, Ed.). Cambridge, MA.

Orfield, G. (2001b). Schools more separate: Consequences of a decade of resegregation. Cambridge, MA: Harvard Civil Rights Project.

Orfield, G. (2009). Reviving the Goal of an Integrated Society: A 21st Century Challenge (Vol. 69). Los Angeles, CA: The Civil Rights Project/Proyecto Derechos Civiles at UCLA.

Puigvert, L., Christou, M., \& Holford, J. (2012). Critical communicative methodology: Including vulnerable voices in research through dialogue. Cambridge Journal of Education, 42(4), 513-526. doi: 10.1080/0305764X.2012.733341

Pulido, C., Elboj, C., Campdepadrós, R., \& Cabré, J. (2014). Exclusionary and Transformative Dimensions Communicative Analysis Enhancing Solidarity Among Women to Overcome Gender Violence.

Qualitative Inquiry, 20(7), 889-894. doi:

$10.1177 / 1077800414537212$

Santiago, C., \& Maya, O. (2012). Segregación escolar del alumnado gitano en España. Córdoba: Federación Nacional de Asociaciones de Mujeres Gitanas KAMIRA \& Fundación Mario Maya. Retrieved from http://federacionkamira.es/wpcontent/uploads/2015/11/Informe-de-Segregación.pdf

Sordé-Martí, T., \& Macías-Aranda, F. (2017). Making Roma Rights a Reality at the Local Level: A Spanish Case Study. In J. Bhabha, A. Mirga, \& M. Matache (Eds.), Realizing Roma Rights (pp. 187-229). Philadelphia, PA: University of Pennsylvania Press.

Sordé, T., Flecha, R., \& Mircea, T. (2013). El pueblo gitano: una identidad global sin territorio [The Roma People. A global identity without territory]. Scripta Nova, 17(427).

The European Union Spanish Presidency. (2010). Conclusions "Science against Poverty" Conference. La Granja, 8-9 April 2010. Brussels: European Union. Retrieved from http://www.scienceagainstpoverty.com/Resources/documentos/result ados/Conclusions_230610.pdf 
Vaillancourt, Y., Ducharme, M. N., Cohen, R., Roy, C., \& Jetté, C. (2001). Social housing: A key component of social policies in transformation: The Quebec experience. Caledon Institute of Social Policy. Ottawa: Caledon Institute of Social Policy.

Valls, R., \& Kyriakides, L. (2013). The power of Interactive Groups: how diversity of adults volunteering in classroom groups can promote inclusion and success for children of vulnerable minority ethnic populations. Cambridge Journal of Education, 43(1), 17-33. doi: 10.1080/0305764X.2012.749213

Vargas, J., \& Gómez, J. (2003). Why Romà do not like mainstream schools: Voices of a people without territory. Harvard Educational Review, 73(4), 559-590. doi: 10.17763/haer.73.4.k6807432592612j3

XTEC - Xarxa Telemàtica Educativa de Catalunya. (2018). Comunitats d'aprenentatge. Retrieved from http://xtec.gencat.cat/ca/projectes/comunitats/

Tania García Espinel es la coordinadora del equipo técnico intercultural del Programa del Pueblo Gitano y de la Innovación Social del Departamento de Trabajo, Asuntos Sociales y Familias, Generalitat de Catalunya. España

Dolores Santiago Santiago es una estudiante gitana del Grado de Trabajo Social, Universidad Rovira y Virgili. España

Manuel García Algar es el co-presidente de CampusRom, la primera Red Gitana Universitaria de Catalunya. España.

Dirección de contacto: tgarciae@ gencat.cat 